\title{
The Effect of Extensive Reading on Vocabulary Knowledge of First Level University Students
}

\section{Campana Dias Diana Carolina \\ Cardenas Moyano Maria Yadira}

Magister en la enseñanza del idioma inglés como lengua extranjera Docente de la Facultad de Salud Pública de la Escuela Superior Politécnica de Chimborazo, Ecuador

\section{Guadalupe Bravo Luis Oswaldo}

Magister en Lingüística Aplicada al Aprendizaje del Inglés, Chimborazo Docente de la Facultad de Salud Pública de la Escuela Superior Politécnica de Chimborazo, Ecuador

\section{Bravo Montenegro Marco Antonio}

Magister en Enseñanza de Inglés como Lengua Extranjera

Docente de la Facultad de Salud Pública de la Escuela Superior Politécnica de Chimborazo, Ecuador

\section{Urena Lara David Antonio}

Licenciado en Ciencias de la Educacion Profesor de Idiomas Ingles Docente de la Facultad de Salud Pública de la Escuela Superior Politécnica de Chimborazo, Ecuador

\section{Abstract}

The objective of the research was to analyze how extensive reading or reading for pleasure can improve the development of vocabulary in a sample of 48 first-level university students of the Universidad Tecnológica Indoamérica in the city of Ambato. It was an experimental, bibliographic, descriptive, field and correlational research. To determine the correlation between the two study variables, a deep and thorough investigative analysis of books, journals and research was carried out. An intervention plan was applied through the use of a handbook of 19 vocabulary activities and to measure the knowledge of the vocabulary in the students, an observation card was used in order to measure attitudes and behavior towards extensive reading. A questionnaire of twelve questions related to word knowledge and the word form with its meaning was used as well. The research contains the sociodemographic statistical analysis of the participants and an analysis of the results of the evaluation of reading knowledge and vocabulary to know the descriptive aspects. Furthermore, the descriptive analysis of the vocabulary 
knowledge assessment from a global point of view and the aspects of word knowledge was carried out; to finally perform the analysis of the presence and degree of correlation between extensive reading and vocabulary knowledge, using the Pearson coefficient. Thus, it allowed to determine that, the existence of the correlation between the two variables investigated. That is to say, the results indicated that the use of extensive reading influences the development of students' vocabulary by raising interest and improving vocabulary.

Keywords: Extensive reading, reading for pleasure, vocabulary, Knowledge, Effect

\section{Introduction}

As English is spoken all over the world, it is acknowledged as the lingua franca or the globalized language. Because of this, better chances are given to people who speak English and thus, there is a necessity to learn it so that people can communicate in a proper way with others and can take these opportunities to grow personally and professionally as well.

Harmer (2001) emphasizes that reading and listening skills imply an active participation from the reader and listener. People use some specialist skills when reading and listening, and a successful understanding of what they are seeing, or hearing depends on a large degree on their proficiency on these specialist skills.

Pigada and Schmitt (2006) conducted a month case study with French explorers; it was an extensive reading programme in order to determine if lexical knowledge could be enhanced. This study assessed a big number of words (133); the study examined the word spelling, grammatical characteristics and their meaning. It used simplified materials, instead of authentic ones and the readers chose 4 books (according to the level) from seventeen titles and they read one book per week. This reading amount is acknowledged as the objective for an extensive reading program to be successful. The measurement used was a one-to-one interview that helped to have a clearer understanding whether learning occurred or not. The results showed that the knowledge of $65 \%$ of the target words improved somehow, the spelling was strongly improved even though the number of exposures was small. Grammatical knowledge and meaning were also improved but not at the same degree. In addition, the study showed that extensive reading enhanced more vocabulary acquisition than prior studies have said.

Additionally, Kargar (2012) mentions that language acquisition and development can be easier by applying extensive reading, which refers to the use of simplified reading material. Thus, Kargar conducted a study with 40 out of 67 Iranian students who failed their first reading course. The students were randomly divided into two groups of 20 students; one was the control 
group and the other one was the experimental one. All the students took a standard reading proficiency test (pre-test) during a 10 weeks program. The experimental group read 10 interesting stories in English meanwhile the control group continued with their normal reading activities. At the end, the two groups took the reading proficiency test, as post-test again, and the results showed that there was an important difference in achievement in the groups. That is to say, extensive reading had a good impact on the proficiency development of low-level EFL students.

A current point in research of reading pedagogy is to know in which setting the readers will learn better: either in a low controlled environment with an enriched atmosphere or in a ruled and instructed atmosphere, focused on the strategies of efficient reading. Krashen's (1993) The Power of reading and Day and Bamford (1998) agreed that extensive reading, which Krashen called free vocabulary reading, is decisive so that students can achieve competency in linguistic, vocabulary, ability in reading, writing and spelling.

Kintsch (1998) states that the process of getting meaning from a text is called reading comprehension. However, the objective is to obtain a general comprehension of the written text instead of gaining the isolated words or sentences meaning. When students understand what they read in the text, they are developing mental models, or meaning representations of the ideas of the text during the reading process. There are two classes of mental models: a textbased model, in which propositions of the text are a mental representation and a situational model that refers to what is understood about the text.

Kelly (1969) recognizes that Harold Palmer applied the term extensive reading in foreign language pedagogy for the first time in his volume 25 Centuries of Language Teaching (p. 131). Palmer was one of the first people that started his studies in language teaching in modern times. He had many skills and one of them was related to terminology. He picked up the word "extensive" from among of several synonyms to transmit an alike idea in his book The scientific study and teaching of languages.

Additionally, Harmer (2007) adds that extensive reading is relevant for the development of students' word recognition and for a general development, as well. It is essential for a good acquisition, not just to read a considerable amount but also to have a well-designed programme that has proper materials, tasks, facilities like handy and long-lasting book libraries and guidance.

The supreme part of vocabulary in first language reading has been well set, the bigger the vocabulary of children, the better their understanding. For instance, Chall (1987) says that each reading achievement study highlights the importance of vocabulary knowledge. Nagy and Hernan (1987) mention it in a similar way; texts are understood better when children know more words. 
This means that, analysing it from a cognitive point of view, there is no significant difference between fluent first and second language reading. Fluent second language reading requires a large amount of vocabulary as fluent first language does. A large number of words is learnt by children when they learn their first language by guessing the meaning in context during reading. Even though, the research on second language reading is not as rich as the research on first language reading is. People who read tons of interesting and diverse material can increase their general vocabulary knowledge. Additionally, second language readers do not have a extensive of oral vocabulary and this is the reason why the texts should not have many unknown and difficult syntactic structures. Thus, the extensive reading approach mentions that students need to read large amount of material, which are easy, diverse and interesting so that they can be able to increase their vocabulary development.

Day and Bamford (1998) also argument that students can have many opportunities to develop this knowledge through an extensive reading approach, which focuses on reading fluency and in the meaning of what is being read. In conclusion, they advise that the development of sight vocabulary, general vocabulary knowledge, and linguistic, is interrelated. It is hard to think that a student who reads extensively can fail in the growth of these knowledge types.

Meara (1980) (as cited in Lightbown and Spada, 2013) highlights that vocabulary learning is a careless point in language learning. In the 70's and 80 's, the researchers paid attention to morphology and syntax because of the manner in which error pattern and the development of sequences of these aspects can show some things related to language acquisition and language. Nevertheless, the things are different now; meanwhile Meara was giving his comments on this, a great movement about research on vocabulary learning started. Moreover, the vocabulary acquisition became one of the main and strong areas in the research of second language acquisition.

The lack of vocabulary knowledge and the lack of use of extensive reading from students sometimes cause difficulties in understanding texts or books. It is important to remember that not having a good motivation and reading texts or books that do not call students attention will cause problems to them because they will not want to read. Therefore, students do not develop their reading fluency because they are just obligated to read what the teachers say or articles their textbooks have. The difficulty of the students to read usually generates a bad or poor impression of them because it might indicate that they have a low reading proficiency and therefore as little literate.

In this respect, it causes weaknesses in the students reading skill and consequently an academic problem due to the fact that students need to master the four language skills at the same degree in order to achieve a B2 level of 
the Common European Framework of Reference (2007) that is established in Ley Orgánica de Educación Superior (2010).

\section{METHODOLOGY}

The research has a qualitative and quantitative approach because the results of the field research were undergone to a numerical analysis using statistics and the data collected was used to prove the hypothesis.

Dawson (2002) mentions that the quantitative research produces statistics applying an extensive survey research. The methods that can be used are structured interviews or questionnaires. He also states that the quantitative research works with more people, but the contact with them within this investigation is faster.

Moreover, he claims that a qualitative research analyses behaviour, attitudes and experiences using methods like focused groups or interviews and it tries to obtain deeper participants' opinions. In this research method, fewer people take part on it.

Indeed, this research method was useful at Universidad Tecnológica Indoamérica because when working with it and with the students using data collection, we were able to analyze conjectures in order to create research questions and to probe the hypothesis previously established.

The use of the bibliographic and documental research in the current project led to examine scientific and trustworthy information comparing the ideas of different authors and taking into consideration the different points of view of each one in order to establish a reliable research of the phenomena under study.

Patton in Cohen, Manion, and Cortese (2006) mention that observational data is appealing because it provides the chance to the researcher to gather information in live, which basically means in live situations. The researcher has the opportunities to see what is happening at the current moment.

Furthermore, Cohen et al. (2006) also state that it gives the opportunity to the researcher to have a clear understanding of things that may be left out with other types or research methods or to reveal things that the participants may not want to talk about.

With the use of this kind of research method in the students of the first level at Universidad Tecnológica Indoamérica, we try to understand the effect of extensive reading in vocabulary knowledge acquisition.

Hernández, Fernández and Baptista, (2014) mention that an experimental research consists in manipulating intentionally an action in order to analyze its possible outcomes. That is to say that two or more independent variables are manipulated in an intentional way (supposed causes), to analyze 
the consequences that this manipulation has upon one or more dependent variables (supposed effects) within a situation that the researcher controls.

Moreover, Cohen et. al (2006) add that the relation between the two variables of the study through the correlational research allows the research to identify the influence of not using extensive reading on vocabulary knowledge. The variables are linked together; hence, if one of them changes, the other one changes too. The established relations between the variables enable to predict the changes that one variable has because of the changes of the other one.

The actual research is descriptive and tries to prove the hypothesis veracity. Salkind (as cited in Bernal 2010) states that the descriptive research describes the characteristics of a phenomenon or situation under study. Additionally, Marin (2008) mentions that this type of research manages to characterize a specific situation or an object of study and points out its properties and peculiarities. It is used to order, group or systematize the objects involved in the investigated work.

The population of the research includes 48 students of the first level (beginners) of courses 1 and 2, at Universidad Tecnológica Indoamérica. They are among 17 to 55 years old. The researcher is the teacher so that it is easier to apply this investigation and the teacher can analyze the data better, as well.

The researcher gave a questionnaire that contains 12 vocabulary words randomly chosen from the glossaries of three books. An observational card was used to determine the attitudes and behaviour towards reading. The students read three books and it is important to mention that they were the ones that chose the books as part of the proposal for this research. This questionnaire has multiple choice statements from which the students needed to choose the correct option. The purpose of this questionnaire is to know and measure the students' vocabulary knowledge.

\begin{tabular}{|c|c|}
\multicolumn{2}{|c|}{ Table 1: } \\
\hline Population & Quantity \\
\hline Students & 48 \\
\hline
\end{tabular}

Made by: Campaña, C. (2017)

The data collection plan of this research includes certain methodological strategies that are related to the research objectives and hypothesis. According to the research method, which is mostly quantitative, the following is taken into consideration:

The technique used was the scientific observation and the instrument used was the structured questionnaire with close-ended questions. Cohen et al. (2006) state that a questionnaire is a suitable instrument to gather information, to give structured and sometimes numerical data and that it can be managed without the researcher. 
This plan considered certain methodological strategies that were suitable to the objectives and to the research method. The plan had correct information avoiding contradictions and it was completed and relevant as well. All the questions were clear in order to have a good organization of the data before the tabulation.

To reach the goal of the current research, we started from the statistical sociodemographic analysis of the participants through the review of the variables of age, gender distribution and the course. Besides, a descriptive statistical analysis was developed of the prevalence and its characteristics.

Then, we proceeded with the analysis of the results obtained from the evaluation of reading knowledge and vocabulary to know the descriptive aspects (means and standard deviations) of the established evaluations, as well as the categorical distribution of the participants.

Subsequently, a second descriptive analysis was carried out. It evaluated the vocabulary knowledge from a global point of view and the aspects of word knowledge: a) word form and b) word meaning. Each one of the exposed dimensions includes a score with ranges between 0 and 6 points. The two factors together make up the global estimate of the variable and these data will fluctuate between the values of 0 to 12 points.

Finally, an analysis of the elements described above was carried out by means of a correlational study to know the presence and degree of correlation between extensive reading and vocabulary knowledge, as well as among its components. Thus, it allowed the confirmation or the rejection of the null hypothesis, both for the presence of correlation and for statistical significance.

\section{ANALYSIS AND INTERPRETATION}

\section{Analysis of Extensive reading}

The evaluation of the extensive reading was done through observation using record cards of the factors: a) interest in books; b) enjoyment; and c): motivation. Each dimension presented encompasses a score of 0 to 2 points. These three factors together form the overall extensive reading estimate. These data fluctuates between values of 0 to 6 points. Higher scores represent a higher presence.

The cut-off point to determine a significant presence corresponds to $70 \%$ of the factors magnitude (1.4 points) and for the overall score (4.2 points). The scores in table 2 indicates the descriptive data encompassing the minimum and maximum scores (Min-Max), the means (Mdn), the standard deviations (SD) and the distribution measures (Kurtosis and Skew). 
Table 2. Descriptive analysis of extensive reading

\begin{tabular}{cccccc}
\hline Factors & Mín. - Máx. & Mean & Dev. & Skew & Kurtosis \\
\hline Interest & $0,40-2,00$ & 1,19 & 0,50 & 0,321 & $-1,464$ \\
Enjoyment & $0,40-2,00$ & 1,14 & 0,45 & 0,517 & $-1,135$ \\
Motivation & $0,60-2,00$ & 1,17 & 0,45 & 0,583 & $-1,074$ \\
\hline Extensive reading & $\mathbf{0 , 8 0}-\mathbf{5 , 9 0}$ & $\mathbf{3 , 5 0}$ & $\mathbf{1 , 3 3}$ & $\mathbf{0 , 4 8 9}$ & $\mathbf{- 1 , 3 1 4}$ \\
\hline \multicolumn{5}{c}{ Note: 48 observations } \\
\hline \multicolumn{5}{c}{ Source: Observational card } \\
& Made by: Campaña Carolina, 2018
\end{tabular}

According to the interest factor the values were Min-Max $=0.4-2.00$ points, with means value of $M d n=1.19$ points and a value of $S D=0.50$. With regard to the distribution measures, skew obtained a value of 0.489 and kurtosis -1.314 .

According to the enjoyment factor, the values were Min-Max $=0.4$ 2.00 points. The values of central tendency measures and dispersion were $M d n$ $=1.14$ points and $S D=0.45$. Moreover, as regards the distribution measures, skew obtained 0.517 and kurtosis obtained -1.135.

Talking about the motivation factor, the descriptive findings show values from 0.60 to 2.00 as the minimum and maximum. Moreover, values of $M d n=1.17$ and $S D=0.45$. While in the distribution scores, skew obtained 0.583 and kurtosis obtained 1.074.

In the overall evaluation of extensive reading, the individual values fluctuated between 0.80 (minimum) and 5.9 (maximum). The central and dispersion scores were $M d n=5.90$ points and $S D=1.33$. Finally, the distribution values were in the skew 0.489 and 1.314 for the kurtosis (see graph $1)$.

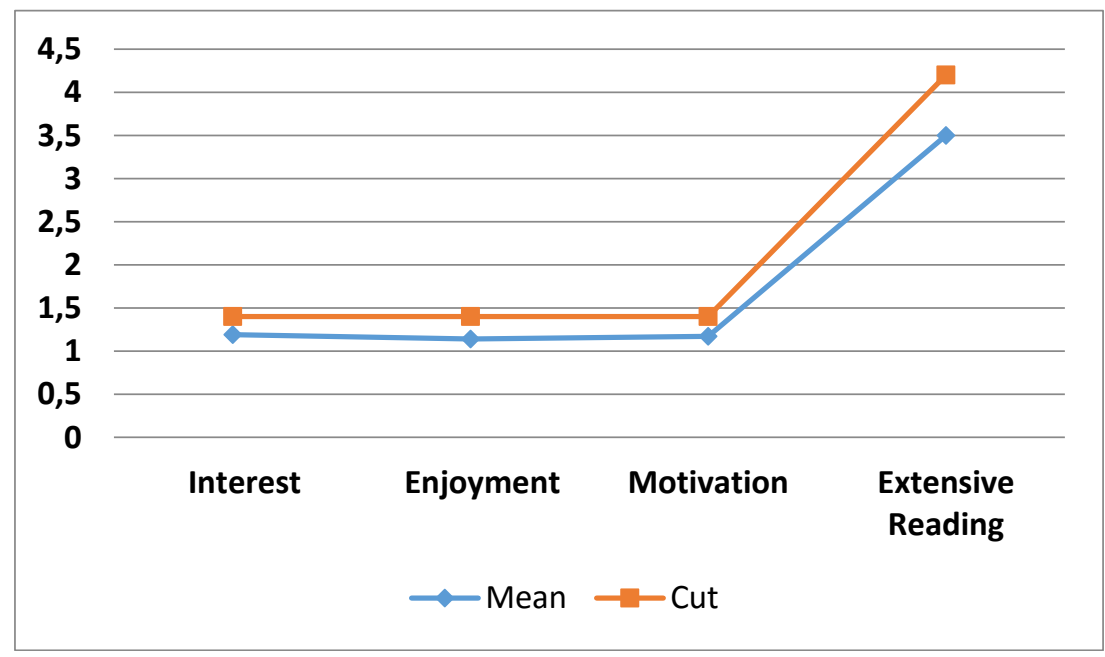




\section{Graph 1. Representation of the descriptive analysis of extensive reading Source: Observational card \\ Made by: Campaña Carolina, 2018}

As you can see in the graph, in all cases, the average scores are below the cut-off point, both the factors as well as the overall score. It means that in a general way, the levels of presence of the extensive reading criteria are low among the participants and do not reach the general estimated criterion of $70 \%$. Thus, it means that the tendency do not display a clear presence of the assessed competence.

\section{Critical analysis}

The categorical analysis allows to interpret the scores obtained among the participants and thus, to know in which group or diagnostic category they are located according to their acquired domains. In the case of extensive reading, three interpretation groups or categories were identified according to the following scores:

- Low level encompasses scores less than two points. They indicate that the domains of extensive reading are insufficient or low. Intervention is required for its domain.

- Medium or moderate level encompasses scores higher than two points up to four points. The presence of the extensive reading domain is limited even though it is not insufficient. These scores are placed in a moderate range of presence. Intervention is required for its improvement.

- High level encompasses scores higher than four points. Extensive reading domain is high and sufficient. The intervention is aimed at maintaining the domain.

The results of the categorical analysis are shown in table 3. It is presented in function of frequency $(f)$ and percentage representation (\%).

Table 3. Categorical analysis of extensive reading

\begin{tabular}{ccc}
\hline Level & Frequency & Percentage \\
\hline Low & 4 & $8,3 \%$ \\
Medium & 27 & $56,3 \%$ \\
High & 17 & $35,4 \%$ \\
\hline \multicolumn{3}{c}{ Note: 48 observations } \\
\hline
\end{tabular}

Source: Observational card

Made by: Campaña Carolina, 2018

The categorical analysis displays that $8.3 \%$ of the participants have a low or deficient extensive reading level, while in the case of the participants with a medium level; they reached a prevalence of $56.3 \%$. Finally, the participants with high level have a 35.4\%. (see graph 12). 


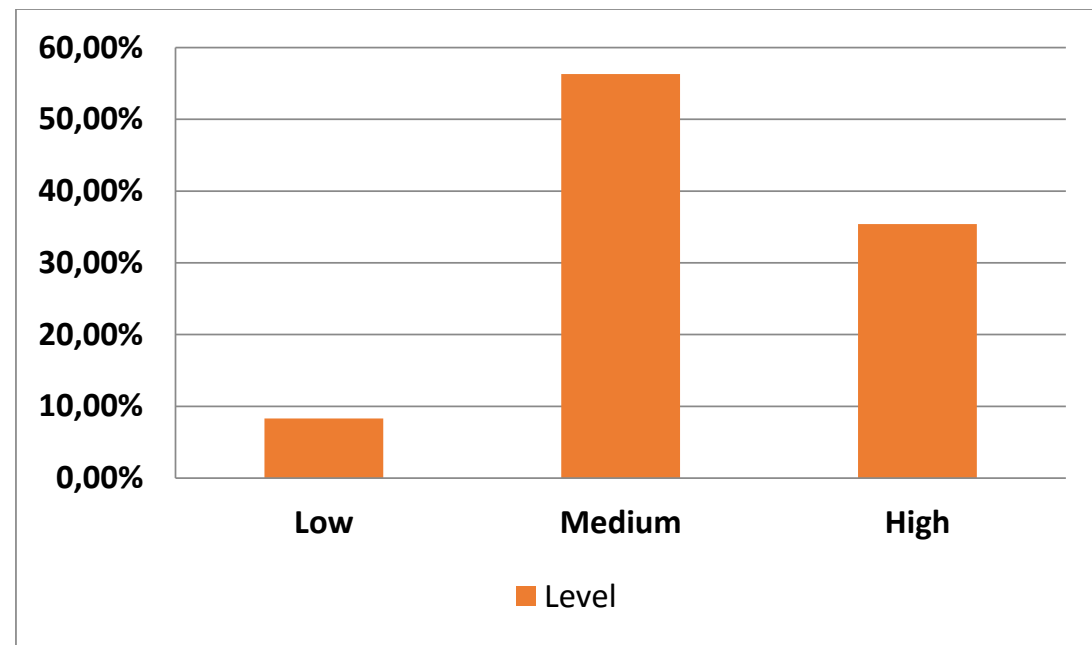

Graph 2. Representation of the categorical analysis of extensive reading Source: Observational card

Made by: Campaña Carolina, 2018

The graph displays the predominance of extensive reading's domain as medium or moderate among the participants of the study. The participants with a high presence of domain 35\% have the second place. Finally, $8 \%$ belongs to the group of participants with a low or insufficient level of domain. This data shows the existing limitations that need to be solved in the future, especially in the participants who belong to the low and medium levels.

\section{Analysis of vocabulary knowledge}

In this second descriptive analysis, the evaluation of vocabulary knowledge included the evaluation of this variable from a global point of view and two aspects of word knowledge: a) word form and b) word meaning. As in the previous analysis, each of the exposed dimensions encompasses a score with ranges between 0 to 6 points. The two factors together form the global estimate of the variable and these data will fluctuate between values of 0 to 12 points. Higher Scores of vocabulary knowledge represent a higher presence of the condition.

The cut-off point to determine significant presence corresponds to $70 \%$ of the magnitude of the factors (1.4 points) and for the overall score (4.2 points). The scores in table 4 display the descriptive data that encompasses the minimum and maximum scores (Min-Max), the means (Mdn), the standard deviations $(S D)$ and the distribution measures (kurtosis and skew). (see table 4) 
Table 4. Descriptive analysis of vocabulary Knowledge

\begin{tabular}{cccccc}
\hline Factors & Mín. - Máx. & Mean & Dev. & Skew & Kurtosis \\
\hline Word form & $1,00-5,00$ & 2,81 & 1,08 & $-0,027$ & $-1,175$ \\
Word meaning & $1,00-6,00$ & 3,48 & 1,69 & $-0,059$ & $-1,575$ \\
\hline Vocabulary knowledge & $2,00-11,00$ & 6,29 & 2,70 & 0,015 & $-1,596$ \\
\hline \multicolumn{6}{c}{ Nota: 48 observations }
\end{tabular}

Source: Questionnaire

Made by: Campaña Carolina, 2018

In the word form factor, the values were Min - Max values $=1.00$ 5.00 points, with mean values of $M d n=2.81$ points and $S D=1.08$. Regarding the distribution measures, the values were -0.027 for the skewness and -1.175 for the kurtosis. While, in word meaning, the results were Min-Max: $=1.00$ 6.00 points. In addition, the value of $M d n=3.48$ points and $S D=1.69$ for the arithmetic mean and for the standard deviation, finally, -0.059 for the skew and -1.575 for the kurtosis.

The vocabulary knowledge findings display values of 2 as minimum and 11 points as maximum. At the level of the mean and standard deviation, results of $M d n=6.29, S D=2.7$ were obtained. Finally, in the case of distribution measures the value for skew was 0.015 and -1.596 for kurtosis (see graph 3).

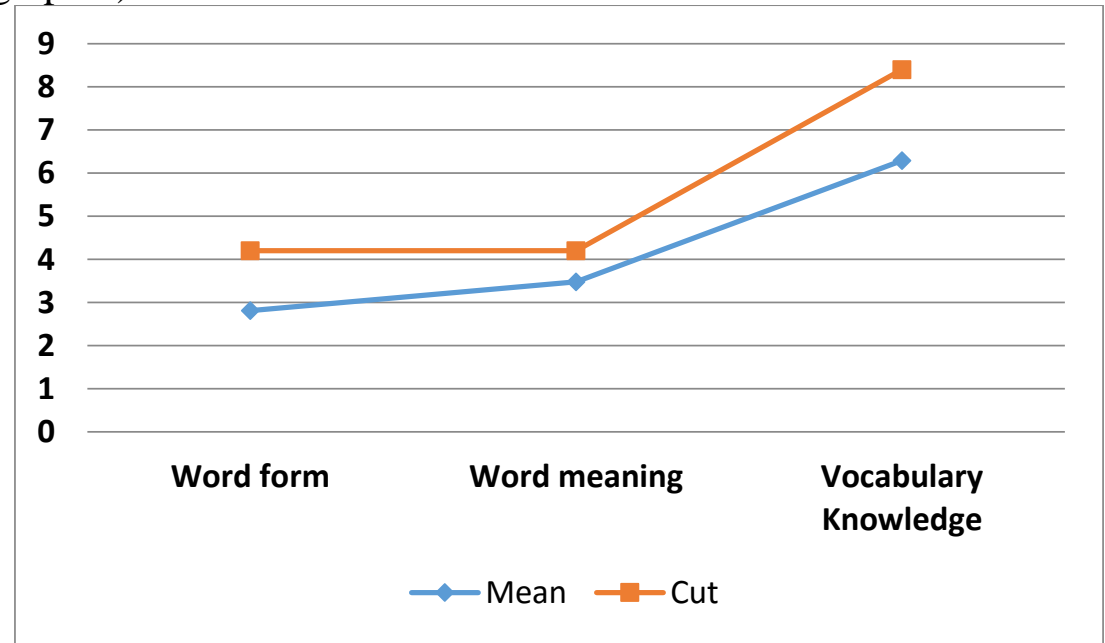

Graph 3. Representation of the critical analysis of vocabulary knowledge Source: Questionnaire

Made by: Campaña Carolina, 2018

The graphic of the descriptive analysis indicates that in the aspects of the vocabulary knowledge as well as in the global score, the presence or the intensity of them are far below from the cut point. That is to say, the perspectives of the competence domain are limited and are insufficient to demonstrate the significant presence of the domain. Thus, in this analysis there 
is already the need to generate improvement processes for the acquisition of these learning elements.

\section{Categorical analysis}

In this second categorical analysis, it can be possible to interpret the scores obtained from the participants and thus, to know in which diagnostic group or category they are placed, based on their acquired domains related to vocabulary knowledge. As in the previous case, three interpretation groups or categories were identified according to the following scores:

- Low level encompasses scores less than four points. They indicate that the domains of vocabulary knowledge are insufficient or low. Furthermore, in these circumstances intervention is required for its domain.

- Medium or moderate level refers to scores higher than four points up to eight points. The presence of the vocabulary knowledge domain is limited, there is an important presence but not significant. Educative intervention is required for its improvement.

- High level encompasses scores higher than eight points. Vocabulary knowledge domain is high, significant and sufficient. Educative intervention is not required, even though it is necessary for maintaining the domain or for acquiring other competences.

The results of the categorical analysis is in table 5, it is presented in function of the frequency $(f)$, and percentage representation $(\%)$.

Table 5. Categorical analysis of vocabulary Knowledge

\begin{tabular}{ccc}
\hline Level & Frequency & Percentage \\
\hline Bajo & 20 & $41,7 \%$ \\
Medio & 7 & $14,6 \%$ \\
Alto & 21 & $43,8 \%$ \\
\hline \multicolumn{3}{c}{ Note: 48 observations }
\end{tabular}

Source: Questionnaire

Made by: Campaña Carolina, 2018

The results show that $41.7 \%$ of the participants have low levels of domain, while $14.6 \%$ have moderate levels and $43.8 \%$ indicate that they have high levels of vocabulary knowledge domain. (see graph 4) 


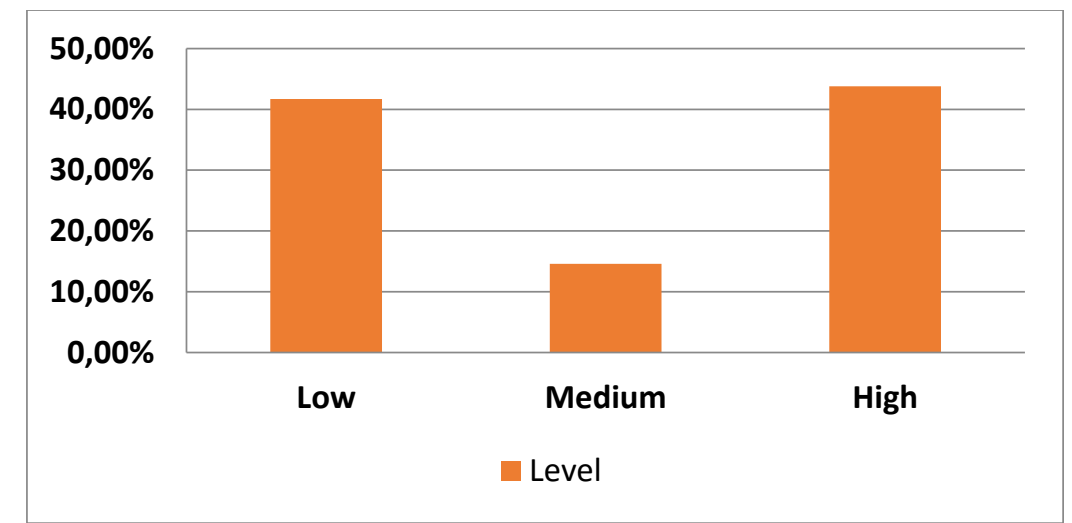

Graph 4. Representation of the categorical analysis of vocabulary knowledge Source: Questionnaire Made by: Carolina Campaña D

The graphical analysis highlights the polarization of the participants in two groups, both at the level of low domain of vocabulary knowledge with approximately $41,7 \%$, and $14.6 \%$ of the moderate domain. They display the tendency of the group to show flaws or limitations of domain. Thus, it requires an educative intervention process for its improvement in the future. Finally, a group of $43.8 \%$ of the participants displays a high and sufficient domain and it represents a significant subgroup of the participants.

\section{Correlation and prediction analysis}

At the end of this analysis, the previously described elements are presented under a correlational study in order to know the presence and degree of correlation between extensive reading and the vocabulary knowledge, as well as between its components. In addition, it helps to discern between the confirmation or rejection of the null hypothesis, both for the presence of correlation and for statistical significance ( $\mathrm{p}<0.05)$.

Pearson product-moment correlation coefficient ( $r$ ) was used in order to develop the correlation analysis. It is the test that allows to identify the presence and degree of correlation between the variables and the components.

The $r$ scores indicate the presence of positive (+) or negative (-) relationship when they move away from 0 and tend to approach to values of 1 and -1 . An acceptable estimate of $r$ corresponds to values above $r>0.2$ and less than $r<0.2$ to determine correlation (see table 6 ). 
Table 6. Correlation analysis between extensive reading and vocabulary knowledge variables

\begin{tabular}{|c|c|c|c|c|c|c|c|}
\hline Factors & IB & $\boldsymbol{E}$ & $M$ & $\begin{array}{c}\text { Extensive } \\
\text { reading }\end{array}$ & $\boldsymbol{W F}$ & $W M$ & Vocabulary \\
\hline Interest in books & 1 &, $845^{* *}$ &, $777^{* *}$ &, $926^{* *}$ &, $685^{* *}$ & ,760** &, $751^{* *}$ \\
\hline Enjoyment & & 1 & $934^{* * *}$ & $974 * *$ &, $680^{* *}$ &, $703^{* *}$ &, $713^{* *}$ \\
\hline Motivation & & & 1 &, $949 * *$ &, $639^{* *}$ &, $662^{* *}$ &, $671^{* *}$ \\
\hline Extensive reading & & & & 1 &, $704^{* *}$ &, $749^{* *}$ &, $752^{* *}$ \\
\hline Word form & & & & & 1 &, $886^{* *}$ &, $957^{* *}$ \\
\hline Word meaning & & & & & & 1 & $983^{* *}$ \\
\hline Vocabulary knowledge & & & & & & & 1 \\
\hline
\end{tabular}

Source: Observational card - Questionnaire Made by: Campaña Carolina, 2018

The correlation analysis indicates the presence of a relationship between the components of the extensive reading and vocabulary knowledge. Thus, the relationship between interest in books and word form questions with $\mathrm{r}=0.685 ; \mathrm{p}<0.001$ is observed; word meaning questions with $\mathrm{r}=0.76 ; \mathrm{p}$ $<0.01$, and with vocabulary knowledge $r=0.751 ; \mathrm{p}<0.001$.

Regarding enjoyment, it correlates with word form questions $r=0.68$; $\mathrm{p}<0.001$; with word meaning questions $\mathrm{r}=0.76$; $\mathrm{p}<0.001$; and with vocabulary knowledge $r=0.713 ; \mathrm{p}<0.001$.

Regarding the motivation component, the correlation with word form questions is $r=0,639 ; p<0.001$; word meaning questions $r=0.662 ; p<0.001$ and with vocabulary knowledge $r=0.621 ; p<0.001$.

Finally, as regards the relationship between the extensive reading and vocabulary knowledge, it was found that there is a relation between both variables with values of $r=0.621 ; p<0.001$.

\section{Prediction analysis}

Because significant and high correlations are found between extensive reading and vocabulary knowledge, a complementary study on this aspect identifies the linear casuistic relationship that can maintain with vocabulary knowledge. It requires the development of an additional study of determination (R2) and its aim is the identification of the predictive power of extensive reading in vocabulary knowledge as we can see in table 16.

Table 7. Prediction analysis of extensive reading upon vocabulary knowledge

\begin{tabular}{|c|c|c|}
\hline Level & Correlation & Determination \\
\hline Interest in books &, $751^{* *}$ & ,564 \\
\hline Enjoyment &, $713^{* *}$ &, 508 \\
\hline Motivation & $671^{* *}$ &, 450 \\
\hline Extensive reading &, $752^{* *}$ & ,566 \\
\hline
\end{tabular}

Source: Observational card - questionnaire

Made by: Campaña Carolina, 2018 
The prediction analysis indicates that extensive reading predicts the changes in the variance in vocabulary knowledge. Thus, it is observed that interest in books predicts $56.4 \%$ the changes in the variance, enjoyment with $50.8 \%$ and motivation with $45 \%$. This is a prediction of extensive reading factors.

Whereas in a global way, it can be considered that extensive reading can predict in $56.6 \%$ the changes of the existing variances in vocabulary knowledge. It can indicate the close relationship between the variables analysed.

\section{Hypothesis verification}

Table 8. Hypothesis verification technical sheet

\begin{tabular}{|c|c|}
\hline & $\begin{array}{l}\text { Null Hypothesis (Ho): There is no correlation between extensive reading and } \\
\text { vocabulary knowledge. }\end{array}$ \\
\hline & $\begin{array}{l}\text { Alternative Hypothesis }\left(\mathbf{H}_{1}\right) \text { : There is correlation between extensive reading and } \\
\text { vocabulary knowledge. }\end{array}$ \\
\hline & $\begin{array}{l}\text { Test of Null Hypothesis (PPMC): Pearson }(r) \text { product-moment correlation } \\
\text { coefficient. }\end{array}$ \\
\hline & $\begin{array}{l}\text { Acceptable value of the test: To be acceptable, The PPMC ( } r) \text { must show that } \\
\text { the variables have a covariation. }(r>0,2) \text {. }\end{array}$ \\
\hline & $\begin{array}{l}\text { Statistical significance (p): The significance must be less than } 5 \%(p<0.05) \text {. The } \\
\text { statistical power indicates that the probability that the data found is due to chance } \\
\text { is less than 5\%. }\end{array}$ \\
\hline & $\begin{array}{l}\text { Results: The } r \text { test indicates values of } 0.752 \text { equivalents to correlation, exceeding } \\
\text { the acceptable value }(r>0.02) \text {. The significance is } p<0.01 \text {, that is to say, the } \\
\text { probability of committing type I error is less than 5\%. }\end{array}$ \\
\hline & $\begin{array}{l}\text { Conclusion: The Ho is rejected because a positive and high correlation exists and } \\
\mathrm{H} 1 \text { is accepted because it marks statistically significant differences and the } \\
\text { probability that these results are due to chance is less than } 1 \%(p<0.01) \text {. }\end{array}$ \\
\hline
\end{tabular}

Made by: Campaña Carolina, 2018

\section{CONCLUSION}

The levels of presence of the extensive reading criteria are low among the participants and do not reach the general estimated criterion of $70 \%$. The categorical analysis displays that $64.6 \%$ of the participants have a low, deficient or medium extensive reading level. Thus, it means that the tendency does not display a clear presence of the assessed competence. Students are not interested enough in the reading material they use in classes and because of that, there is a lack of enjoyment and motivation while reading.

The presence or the intensity of the two aspects of vocabulary knowledge, word form and word meaning, are far below from the cut point. That is to say, the perspectives of the competence domain are limited and are insufficient in order to demonstrate the significant presence of the domain. The critical analysis displays that $56.3 \%$ of the participants have a low, 
deficient or medium levels of vocabulary knowledge domain. They display flaws or limitations of domain.

After trying to correlate the two variables using suitable strategies to enhance vocabulary knowledge through extensive reading, the correlation analysis indicates the presence of a relationship between the components of the extensive reading and vocabulary knowledge. There is a relation between both variables with values of $\mathrm{r}=0.621, \mathrm{p}<0.001$. The prediction analysis indicates that extensive reading predicts the changes in the variance in vocabulary knowledge. Thus, in a global way, it can be considered that extensive reading can predict in $56.6 \%$ the changes of the existing variances in vocabulary knowledge. Finally, it indicates the close relationship between the variables analysed as well. Thus, the use of a vocabulary handbook has a positive effect upon the development of vocabulary knowledge.

\section{RECOMMENDATIONS}

It is suggested that teachers implement extensive reading besides the ones they utilize during their classes so that learners can enjoy and be motivated while reading. It is relevant that students can also choose the reading material for their reading lessons.

It is highly important that teachers help learners to enhance their domain or limitations of vocabulary knowledge. Teachers must apply an educative intervention and give students as many opportunities as possible for its improvement.

Because there is a relationship between the components of extensive reading and vocabulary knowledge; it is advisable that teachers utilize extensive reading material and a handbook created by the author that contains vocabulary activities to help students to enhance their vocabulary knowledge. In this way, learners can work with this material in classes and outside them as well. Thus, they will enjoy the reading and will see their lessons as a meaningful process that can lead them to acquire more vocabulary.

\section{References:}

1. BERNAL, César A. (2010). Metodología de la Investigación. Tercera edición. Pearson, Educación: Colombia.

2. CHALL, J. S. 1983a.Stages of Reading Development. New York: McGraw-Hill.

3. COHEN, L., MANION, L. \& MORRISON, K. (2006). Research Methods in Education. 6th Ed. New York: Routledge Falmer.

4. DAY, R., \& Bamford, J. (1998). Extensive reading in the second language classroom. New York: Cambridge University Press.

5. HARMER, J. (2001). The practice of language teaching. Longman. 
6. HARMER, J. (2007). The practice of English language teaching. White Plains, NY: Longman.

7. HERNÁNDEZ Sampieri, R., FERNÁNDEZ Collado, C., \& BAPTISTA Lucio, P. (2014). Metodología de la investigación: Roberto Hernández Sampieri, Carlos Fernández Collado y Pilar Baptista Lucio (6a. ed. --.). México D.F.: McGraw-Hill.

8. KARGAR, A. A. (2012). The efficiency of extensive reading project (ERP) an Iranian EFL context. Academy Publisher, 165-169. Retrieved from https://search.proquest.com/openview/66fd6dfe 36e2bc1898c6 ddaf87cc240 9/1?pq-origsite $=$ gscholar $\& \mathrm{cbl}=2026476$

9. KELLY, L. G. (1969). 25 centuries of language teaching. Rowley, MA: Newbury House

10. KINTSCH, W. (1998). Comprehension: A paradigm for cognition. Cambridge: University Press.

11. KRASHEN, S. D. (1993). The power of reading: Insights from the research. Englewood, Colo: Libraries Unlimited.

12. LEY ORGÁNICA DE EDUCACIÓN SUPERIOR, (2010). Registro Oficial. [DX Reader version]. $\quad$ Retrieved from http://www.fci.utm.edu.ec:81/doc/loes.pdf

13. MEARA, P. (1980). Vocabulary acquisition: A neglected aspect of language learning. Language teaching and linguistics Abstracts.

14. NAGY, W. E., ANDERSON, R. C., \& Herman, P. A. (1987). Learning Word Meanings From Context During Normal Reading. American Educational Research Journal, 24(2), 237-270.

15. PIGADA, M., \& Schmitt, N. (2006). Vocabulary acquisition from extensive reading. A case study. Reading in a foreign language. Retrieved from http://nflrc.hawaii.edu/rfl/ 